\title{
La colonografia tomográfica sin preparación colónica podría representar una alternativa de rastreo del cáncer de colon
}

Tomographic colonography without bowel preparation could represent an alternative to colorectal cancer screening

\section{Objetivos}

Establecer las características operativas de la colonografía tomográfica (CT) sin preparación purgante, para la detección de pólipos adenomatosos mayores a $6 \mathrm{~mm}$.

\section{Diseño, población y lugar}

Cohorte prospectiva (cuatro centros médicos de EE.UU.). A todos los pacientes que estaban en lista de espera para videocolonoscopía (VCC) de rastreo se les ofreció realizar además previamente una colonografía tomográfica de 16 o 64 pistas sin preparación colónica $(n=618)$.

\section{Descripción de la prueba y del estándar de referencia} Colonografía tomográfica: durante los tres días previos al procedimiento se indicó a los pacientes ingerir un preparado destinado a marcar las heces para poder ser identificadas a través de un software y luego detectar posibles pólipos. Los resultados fueron evaluados por tres radiólogos entrenados.

Videocolonoscopía: requería preparación colónica. Se utilizó técnica habitual con sedación.

Estándar de referencia: a medida que el gastroenterólogo retiraba el colonoscopio, se le proporcionaban los hallazgos de
Zalis ME y col. Ann Intern Med. 2012;156:692-702 la CT de ese segmento y volvía a examinar esa sección de colon, esos resultados fueron tomados como el estándar de referencia*.

Encuesta sobre la experiencia de los pacientes: se indagó acerca del disconfort, síntomas gastrointestinales, dificultad para completar el estudio y preferencias de técnica.

\section{Medición de resultados principales}

Se calcularon la sensibilidad*, especificidad ${ }^{*}$, valores predictivos positivos ${ }^{*}$ y negativos ${ }^{*}$ y coeficientes de probabilidad positivos $^{*}$ y negativos ${ }^{*}$, comparando CT y VCC contra el estándar de referencia.

\section{Resultados principales}

No se observaron diferencias en la sensibilidad para detectar pólipos mayores a $6 \mathrm{~mm}$. Hubo diferencias estadísticamente significativas en la especificidad entre ambas técnicas para la detección de lesiones mayores de 6 y 8 mm (>6mm p=0,001; $>8 \mathrm{~mm} \mathrm{p}=0,02$ ) (Tabla 1)

Los resultados de la encuesta realizada a los pacientes favorecieron a la CT en relación a sintomatología y confort $(p=<0,001)$; el $62 \%$ de los pacientes refirieron preferir la CT por sobre la VCC $(p=<0,001)$.

Tabla 1. Características operativas de ambas técnicas según el tamaño de pólipo.

\begin{tabular}{|c|c|c|c|c|c|c|}
\hline \multirow{3}{*}{$\begin{array}{l}\text { Caracteristicas } \\
\text { (IC 95\%) }\end{array}$} & \multicolumn{6}{|c|}{ Tamaño de la lesión según estándar de referencia } \\
\hline & \multicolumn{2}{|c|}{ Mayor a $6 \mathrm{~mm}$} & \multicolumn{2}{|c|}{ Mayor a 8 mm } & \multicolumn{2}{|c|}{ Mayor a $10 \mathrm{~mm}$} \\
\hline & CT & VCC & CT & veC & CT & VCC \\
\hline Sensibilidad & $0,59(0,5$ a 0,7$)$ & $0,76(0,6$ a 0,8$)$ & $0,70(0,5$ a 0,8$)$ & $0,88(0,7$ a 0,9$)$ & $0,91(0,7$ a 0,99$)$ & $0,96(0,8 \mathrm{a} 1)$ \\
\hline Especificidad & $0,88(0,8$ a 0,9$)$ & $0,94(0,9$ a 0,96$)$ & $0,86(0,8$ a 0,9$)$ & $0,91(0,8$ a 0,9$)$ & $0,85(0,8$ a 0,9$)$ & $0,89(0,8$ a 0,9$)$ \\
\hline VPP & $0,42(0,3$ a 0,5$)$ & $0,64(0,5 \mathrm{a} 0,7)$ & $0,26(0,2$ a 0,4$)$ & $0,40(0,3$ a 0,5$)$ & $0,19(0,1 \mathrm{a} 0,3)$ & $0,24(0,2$ a 0,35$)$ \\
\hline VPN & $0,94(0,9$ a 0,96$)$ & $0,97(0,9$ a 0,98$)$ & $0,98(0,9$ a 0,99$)$ & $0,99(0,98$ a 1) & $1,00(0,99$ a 1$)$ & $1,00(0,9 \mathrm{a} 1)$ \\
\hline $\mathrm{CPP}$ & $5,09(3,8$ a 6,9$)$ & $12,96(9$ a 18,7$)$ & $5,07(3,8$ a 6,8$)$ & $9,51(7,2$ a 12,6$)$ & $6,2(4,9$ a 7,8$)$ & $8,43(6,6$ a 10,8$)$ \\
\hline CPN & $0,46(0,35$ a 0,6$)$ & $0,26(0,2$ a 0,4$)$ & $0,35(0,2$ a 0,6$)$ & $0,14(0,06$ a 0,3$)$ & $0,11(0,03$ a 0,4$)$ & $0,05(0,01 \mathrm{a} 0,35)$ \\
\hline
\end{tabular}

$\mathrm{VPP}=$ valor predictivo positivo; $\mathrm{VPN}=$ valor predictivo negativo: $\mathrm{CPP}=$ coeficiente de probabilidad positivo; $\mathrm{CPN}$ : coeficiente de probabilidad negativo .

Conclusión

La CT mostró una adecuada sensibilidad y especificidad en pólipos mayores a $10 \mathrm{~mm}$ produciendo menos disconfort que la VCC, lo que podría aumentar su adherencia.

\section{Comentario}

Datos actuales colocan al cáncer de colon en el segundo lugar en relación a la mortalidad por causas oncológicas en EEUU1. Dadas sus características intrínsecas, es una patología ideal para ser rastreada en la población general. Sin embargo, la adherencia global al rastreo no supera el $60 \%$ bajando incluso a valores de $38 \%$ para $\mathrm{VCC}^{2}$. Según algunos estudios la principal causa de la baja adherencia sería la preparación colónica previa ${ }^{3}$. Esto podría otorgarle relevancia a técnicas como la CT. Sin embargo, hay que recordar que al igual que otras técnicas "intermedias", como la determinación de sangre oculta en materia fecal, de detectarse una lesión sospechosa se indicará a continuación una VCC (con preparación colónica) que permita resecar la misma y determinar su histopatología. Asimismo, dada su menor especificidad en pólipos $>10 \mathrm{~mm}$, la tasa de falsos positivos con CT no sería la óptima para una estrategia de rastreo. Por último, hay que tener en cuenta el hallazgo de lesiones incidentales, dado que se tomografía todo el abdomen.

\section{Conclusión del comentador}

Aunque la CT no reemplace estrategias diagnósticas anteriores, pareciera ofrecer una alternativa para aquellos pacientes que rechacen la VCC, al menos de manera inicial.

Santiago Esteban [ Servicio de Medicina Familiar y Comunitaria. Hospital Italiano de Buenos Aires. santiago.esteban@ hiba.org.ar]

Esteban S. La colonografía tomográfica sin preparación colónica podría representar una alternativa de rastreo del cáncer de colon. Evid Act Práct Ambul. Jul-Set 2012;15(3):115. Comentado de: Zalis ME et al. Diagnostic accuracy of laxative free computed tomographic colonography for detection of adenomatous polyps in asymptomatic adults. Ann Intern Med. 2012;156: 692-702. PMID: 22586008.

\section{Referencias}

1.Jemal A, y col. Cancer Statistics, 2008. CA: A Cancer Journal for Clinicians .2008;58(2):71-96.

2. Inadomi J, y col. Adherence to colorectal cancer screening: a randomized clinical trial of competing strategies. Archives of internal medicine. 2012;172(7):575-582.

3. Beebe $\mathrm{T}$ y col. Assessing attitudes toward laxative preparation in colorectal cancer screening and effects on future testing: potential receptivity to computed tomographic colonography. Mayo Clinic proceedings. Mayo Clinic. 2007;82(6):666-671 